International Journal of Biological Sciences

ISSN 1449-2288 www.biolsci.org 2008 4(3):150-160

Research Paper

CIvyspring International Publisher. All rights reserved

\title{
Evaluation of the eifects of a new drug candidate (GEMSP) in a chronic EAE model
}

\author{
A. Mangas ${ }^{1,2}$, R. Coveñas ${ }^{2}$, D. Bodet ${ }^{1}$, M. de León 3 , S. Duleu ${ }^{3}$ and M. Geffard 4 \\ 1. Gemac S.A., Immunochemistry Department, Saint Jean d'Illac, France \\ 2. Institute of Neurosciences of Castilla y León (INCYL), Laboratory of Neuroanatomy of the Peptidergic Systems, \\ Salamanca, Spain \\ 3. IDRPHT, Talence, France \\ 4. IMS Laboratory, ENSCPB, Pessac, France
}

Correspondence to: Arturo Mangas, PhD, University of Salamanca, Institute of Neurosciences of Castilla y León (INCYL), Laboratory of Neuroanatomy of the Peptidergic Systems, C/ Pintor Fernando Gallego 1, 37007 Salamanca, Spain. Tel: 34-923-294400 extension 1856; Fax: 34-923-294549; E-mail: mangasam@usal.es

Received: 2008.01.11; Accepted: 2008.05.19; Published: 2008.05.22

Chronic Experimental Autoimmune Encephalomyelitis (EAE) was induced in rats to evaluate a new drug candidate (GEMSP) for the treatment of multiple sclerosis. This work is a part of preclinical studies on GEMSP, which is made up of fatty acids, vitamins and amino acids or their derivatives; all these compounds were linked to Poly-L-Lysine. In order to evaluate the effects of GEMSP, animals were divided into three experimental groups: 1) EAE rats treated with GEMSP; 2) EAE rats treated with $\mathrm{NaCl}$; and 3) non-EAE rats. Using immunocytochemical techniques with a pan-leukocyte marker (anti-CD 45), differential leukocyte infiltration was compared in the central nervous systems of the different experimental groups. Antibodies directed against a component of GEMSP, the conjugated methionine, were used in all three groups. We found that: 1) GEMSP was effective in abolishing EAE. The crises and clinical scores were completely abolished in the animals of the first group, but not in the animals belonging to the second group; 2) the degree of leukocyte infiltration varied, depending on the different EAE stages, but was not related to the clinical score; and 3) after using anti-conjugated methionine antibodies, we observed immunoreactivity only in the motoneurons of the ventral horn of the spinal cord in the animals of the first group. This immunoreactivity was not found in the animals of the second or third groups. No methionine immunoreactivity was found in the brain. Our results suggest that GEMSP may be a potential drug candidate against the pathogenic processes involved in multiple sclerosis, inhibiting EAE episodes and brain leukocyte infiltration. Our results also show that one component of GEMSP, the methionine compound, is stored inside motoneurons. The possible physiological actions of GEMSP on spinal cord motoneurons are discussed.

Key words: EAE-MS, leukocyte infiltration, CD 45, spinal cord motoneurons, conjugated methionine, immunocytochemistry.

\section{Introduction}

Multiple Sclerosis (MS) is a chronic and progressive inflammatory autoimmune disease of the central nervous system (CNS) whose aetiology remains unknown. During the course of the disease, patients present focal leukocyte infiltration, which produces inflammation followed by demyelization and, as the disease progresses, patients develop increasing handicaps. However, currently tested and approved drugs for the medical treatment of MS have failed to change the course of the illness. In addition, such drugs elicit well-reported side effects [1].

Experimental autoimmune encephalomyelitis (EAE) is an autoimmune inflammatory disease of the rodent CNS that is widely used for the evaluation of different drugs ( $\beta$ interferon (IFN- $\beta$ ), glatiramer acetate (GA), laquinimod, and linomide) [2-7]. Among the multiple possibilities and variations, there are two classical models of EAE: a) acute EAE, characterized by a clear EAE crisis accompanied by inflammation of the CNS, with a relatively short duration and nearly complete recovery of the animals (see [8]); and b) chronic EAE, in which the animals present clinical signs for at least two months with several EAE crises, and in general, they remain with a handicap; a sign of irreversible damage to the CNS. The main advantages of this latter model are that it appears to mimic the pathogenesis of MS; the disease changes over long periods of time; and no recovery of the clinical signs appears due to the demyelization processes.

Brain leukocyte infiltration is related to inflammation, and has previously been evaluated in EAE models in which animals were treated with different drugs: IFN- $\beta[3,6,9]$, Laquinimod [5], Linomide [10] or dexamethasone [11]. Currently 
approved therapies (IFN- $\beta$ and GA) are assumed to exert their effects on the Th1/Th2 phenotype ratio of T-cells [12], although MS inflammatory lesions do not predict later changes in impairment or disability [13]. This means that treatment focused exclusively on inflammation is not appropriate, and this should encourage the scientific community to search for new perspectives and new treatments for MS.

In an acute EAE model, we previously presented the drug studied here, designated GEMSP, as a new candidate for MS treatment. In that acute model, GEMSP inhibited both the EAE episode and brain leukocyte infiltration [8]. However, despite the demonstrated beneficial effects of GEMSP in the acute animal model of EAE, to date the presence of the drug in the rat central nervous system has not been confirmed. Moreover, no studies have been carried out on the actions of GEMSP in chronic EAE models. To date, no side effects have been reported for GEMSP either in an open clinical trial or in experimental animals, since GEMSP does elicits neither biological or haematological nor hepatic side effects [8]. It should be noted that GEMSP (used in the animal models) and GEMSEP1, used in the clinical trial, do not have different components, and were designated thus only in order to differentiate between the data obtained in animal models and in clinical trials.

Thus, using a chronic EAE model [14], characterized by a long-term disease and currently accepted by the scientific community, the aims of the present study were to test the effects of GEMSP on EAE episodes and CNS leukocyte infiltration, as well as to demonstrate that some components of GEMSP are stored inside neurons.

\section{MATERIALS AND METHODS}

\section{Animals}

Thirty-one Lewis 1A female rats aged 10-11 weeks (weight around $190 \mathrm{~g}$ ) obtained from CERJ Janvier (France) were used in this study. The animals were kept under standardized light and temperature conditions and had free access to food and water. They remained in their cages for at least two weeks before the experiments (day 14, D -14) (see Figure 1).

\section{Induction of EAE}

Chronic EAE induction was performed on day 0 (D0, see experimental procedure, depicted in Figure 1) in groups 1 and 2 (see below). Each rat from these groups was immunized with a $250 \mu$ l-solution containing: $50 \mu \mathrm{g}$ of myelin oligodendrocyte glycoprotein (MOG), produced and purified at the laboratory of Professor Villoslada (Pamplona, Spain) (see [15]), and complete Freund's adjuvant (ACF), purchased from Sigma, to which $200 \mu \mathrm{g}$ of heat-inactivated Mycobacterium tuberculosis H37RA (Difco) had been added. The rats were anaesthetized with isofluorane and the solution was once injected intradermally at the base of the tail.

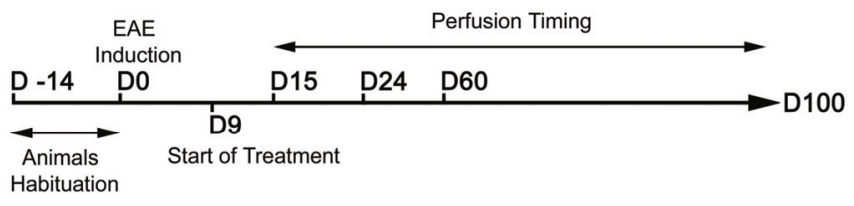

FIGURE 1. Experimental procedure. The main experimental processes are represented in this figure for a better appreciation of the timing of each manipulation (induction, beginning of treatment, and perfusions). D: days; D -14: arrival date of the animals; D0: date of model induction; D9: start of treatment; D15, D24, D60 and D100: days on which animals were perfused.

\section{Animal Groups and Clinical Evaluation of EAE}

Two groups of animals were immunized on D0: 1) EAE-immunized rats treated with GEMSP $(n=10)$ and 2) EAE-immunized rats treated with $\mathrm{NaCl}(\mathrm{n}=18)$. A third group of animals was not immunized (non-EAE-immunized rats treated with $\mathrm{NaCl}$ ) (control) $(n=3)$. The study, using the three groups, was repeated twice (first experiment, $\mathrm{n}=14$; second experiment, $\mathrm{n}=17$ ) and the same batches of GEMSP and MOG were used in both cases. We found no difference between the first and second experiments.

The animals were weighed and scored according to a previously described scale [14] 6 days/week throughout the experiments by two alternating investigators. The neurological signs of EAE, which started around day fifteen after its induction (D15, see Figure 1), were assessed and scored using the following scale: 0 , no signs; 1 , tail weakness or tail paralysis; 2, hind leg paraparesis or hemiparesis; 3, hind leg paralysis or hemiparalysis; 4, complete paralysis (tetraplegy).

Treatment of the animals began nine days after the induction of chronic EAE (D9, see Figure 1). All animals received a single daily subcutaneous injection of their respective solution after D9. Thus, the animals in group 1 were treated with $7.5 \mathrm{mg}$ of GEMSP per day (dissolved in $0.5 \mathrm{ml}$ of a $\mathrm{NaCl}$ solution) and the animals in groups 2 and 3 received $0.5 \mathrm{ml}$ of a $\mathrm{NaCl}$ solution per day. The experimental design, protocols, and procedures used in this work were performed under the guidelines of the ethics and legal recommendations of Spanish, French, and European legislation. This work was also approved by the Bioethics Committee of the University of Salamanca (Spain). 


\section{Synthesis of the Drug Candidate and Immunocytochemistry}

GEMSP was synthesized according to patent numbers 6114388 (USA) and 792167 (EU). This drug is a functional polycomplex in which different compounds are linked to Poly-L-Lysine via glutaraldehyde, glutaric anhydride, or amide bonds (see [8]). The different families of components included in GEMSP had different properties and concentrations. The concentrations of each component are detailed in Table 1.

TABLE 1. GEMSP constituents. Different families of GEMSP constituents with the respective concentration of each compound in the solution used for treating the animals. This treatment took place under identical conditions throughout the experiments, using the same lot number. Lyophilised GEMSP was reconstituted with purified water before subcutaneous injection. AA: amino acids; M: molarity; PL: Poly-L.Lysine

\begin{tabular}{|c|c|c|}
\hline $\begin{array}{l}\text { Families of } \\
\text { Compounds }\end{array}$ & $\begin{array}{l}\text { Different Constituents of } \\
\text { GEMSP Linked to PL }\end{array}$ & $\begin{array}{c}\text { Final } \\
\text { Concentration } \\
\text { of GEMSP } \\
\text { (M) }\end{array}$ \\
\hline \multirow[t]{11}{*}{ Fatty Acids } & Azelaic Acid - PL - Oleic Acid & $1.00 \mathrm{E}-03$ \\
\hline & $\begin{array}{c}\text { Azelaic Acid - PL - Palmitoleic } \\
\text { Acid }\end{array}$ & $1.00 \mathrm{E}-03$ \\
\hline & $\begin{array}{c}\text { T-T-Farnesyl-L.Cysteine - PL - } \\
\text { Oleic Acid }\end{array}$ & $1.00 \mathrm{E}-03$ \\
\hline & Oleic Acid - PL - Palmitic Acid & $1.00 \mathrm{E}-03$ \\
\hline & Oleic Acid - PL - Myristic Acid & $1.00 \mathrm{E}-03$ \\
\hline & Oleic Acid - PL - Linoleic Acid & $1.00 \mathrm{E}-03$ \\
\hline & Oleic Acid - PL - Thioctic Acid & 2.00E-04 \\
\hline & Cholesterol -PL - Oleic Acid & $1.00 \mathrm{E}-03$ \\
\hline & Linoleic Acid - PL & $1.00 \mathrm{E}-03$ \\
\hline & $\begin{array}{c}\text { Oleic Acid - PL - Palmitoleic } \\
\text { Acid }\end{array}$ & 1.00E-03 \\
\hline & $\begin{array}{c}\text { T-T-Farnesyl-L.Cysteine - PL - } \\
\text { Palmitic Acid }\end{array}$ & $2.00 \mathrm{E}-04$ \\
\hline \multirow[t]{2}{*}{ Antioxidants } & a-Tocopherol-succinate - PL & $2.00 \mathrm{E}-04$ \\
\hline & Ascorbic Acid - PL & 2.00E-04 \\
\hline \multirow[t]{6}{*}{ AA and Derivatives } & $\begin{array}{l}\text { L.Methionine - Reduced } \\
\text { Glutaraldehyde - PL }\end{array}$ & 2.00E-04 \\
\hline & $\begin{array}{l}\text { L.Cysteine - Reduced } \\
\text { Glutaraldehyde - PL }\end{array}$ & 2.00E-04 \\
\hline & $\begin{array}{l}\text { Taurine - Reduced } \\
\text { Glutaraldehyde - PL }\end{array}$ & 2.00E-04 \\
\hline & $\begin{array}{l}\text { Histamine - Glutaric } \\
\text { Anhydride - PL }\end{array}$ & 4.00E-04 \\
\hline & $\begin{array}{l}\text { L.Histidine - Glutaric } \\
\text { Anhydride - PL }\end{array}$ & 4.00E-04 \\
\hline & $\begin{array}{l}\text { 5-Methoxytryptamine - } \\
\text { Glutaric Anhydride - PL }\end{array}$ & 4.00E-04 \\
\hline
\end{tabular}

\section{Immunocytochemistry}

The animals were deeply anaesthetized with urethane $(1 \mathrm{~g} / \mathrm{kg}$, intraperitoneal), heparinized, and perfused via the ascending aorta with $50-100 \mathrm{ml}$ of cold physiologic saline $(0.9 \% \mathrm{NaCl})$ and with $500 \mathrm{ml}$ of cold $4 \%$ paraformaldehyde in $0.1 \mathrm{M}$ phosphate-buffer (PB), $\mathrm{pH} 7.2$ (10 min). Perfusions were carried out on different days (D15, D24, D60 and D100; see Figure 1) to check the evolution of CNS leukocyte infiltration. After perfusion, the CNS was dissected out and processed as previously described for immunocytochemistry (see [8, 16-18]). In order to avoid possible interference with endogenous peroxidase, free-floating sections were pre-treated with a solution containing methanol and $\mathrm{H}_{2} \mathrm{O}_{2}(2 / 1)$ for $30 \mathrm{~min}[8,16-19]$. Then, the sections were washed for $30 \mathrm{~min}$ (at room temperature, "RT") in $0.15 \mathrm{M} \mathrm{PB}$ saline (PBS) ( $\mathrm{pH}$ 7.2), after which they were pre-incubated for $30 \mathrm{~min}$ (RT) in PBS containing 10\% of normal horse serum and $0.3 \%$ of Triton X-100 (PBSt). The sections were then incubated overnight at $4^{\circ} \mathrm{C}$ in PBSt containing monoclonal anti-rat CD 45 antiserum (purchased from Serotec or Pharmingen and diluted $1 / 500$ ) to demonstrate leukocyte infiltration and anti-rabbit conjugated methionine antiserum (purchased from Gemacbio, France, and diluted $1 / 1,000)$ to demonstrate GEMSP. Sections were then washed in PBS (30 min, RT), incubated for $1 \mathrm{~h}$ (RT) with biotinylated anti-mouse or anti-rabbit immunogammaglobulin (Vector and Serotec), diluted 1/200 in PBSt, and washed with PBS (30 min) at RT. Following this, sections were incubated for $1 \mathrm{~h}$ (RT) with 1/100 diluted avidin-biotin-peroxidase complex (Vectas'tain) in PBSt. The sections were then washed again in PBS for $30 \mathrm{~min}$ (RT) and Tris- $\mathrm{HCl}$ buffer ( $\mathrm{pH}$ 7.6) (10 min; RT). Tissue-bound peroxidase was developed with $\mathrm{H}_{2} \mathrm{O}_{2}$ using 3, 3' diaminobenzidine as chromogen. The sections were then rinsed with PBS and coverslipped with PBS/Glycerol (1/1).

The specificity of the tissue immunoreactivity has already been tested for antibodies directed against CD 45 (see [8]). As previously reported, identical results were obtained after application of the immunocytochemical technique using the anti-CD 45 purchased from Pharmingen and Serotec (see [8]). Antibodies directed against the conjugated methionine were obtained after several immunizations with the methionine-glutaraldehyde (G)-bovine serum albumin (BSA) immunogen $(250 \mu \mathrm{g})$ in a solution of complete or incomplete Freund's adjuvant $(0.5 \mathrm{ml})$ and $\mathrm{NaCl}(9$ $\mathrm{g} / \mathrm{l})(0.5 \mathrm{ml})$. The serum obtained was preabsorbed with (BSA-G) and purified. Regarding the specificity of the anti-conjugated methionine antiserum, the following histological controls were carried out: a) omission of the primary antiserum in the first incubation bath; and b) preabsorption of the anti-conjugated methionine antibody with conjugated methionine $(300 \mu \mathrm{g}$ of methionine-G-BSA per $\mathrm{ml}$ of diluted antiserum) or with methionine-G-PL (300 $\mu \mathrm{g}$ of GEMSP per $\mathrm{ml}$ of diluted antiserum). No residual immunoreactivity was found in any case. In addition, the absence of immunoreactivity for conjugated 
methionine in animals not treated with GEMSP (second and third groups) and the presence of such immunoreactivity in animals of the first group (rats treated with GEMSP) also indicated the specificity of the immunoreactivity. The ELISA tests carried out revealed that the signal observed in the tissue was specific for conjugated methionine (see Table 2, taken from the data sheet of Gemacbio Laboratories).

TABLE 2. Using competition ELISA tests (data obtained from the data sheet of GEMACBIO), cross-reactivity was calculated from the displacement curves at half-displacement: the best recognized was methionine-Gc, whose concentration was divided by the concentration of each of the other conjugates. Gc: conjugated via glutaraldehyde.

\begin{tabular}{|c|c|}
\hline Compound & Cross-reactivity ratio \\
\hline L. Methionine-Gc & 1 \\
\hline Homocysteine-Gc & $1 />1,000$ \\
\hline Homocysteic acid-Gc & $1 />10,000$ \\
\hline Cystathionine-Gc & $1 />10,000$ \\
\hline L. Cysteine-Gc & $1 />10,000$ \\
\hline L. Glutamate-Gc & $1 />10,000$ \\
\hline
\end{tabular}

\section{Image Processing}

Finally, the stereotaxic atlas of Paxinos and Watson [20] was used for nomenclature and mapping. Photomicrographs were obtained with an Olympus DP-50-CU digital camera attached to a Leica microscope. To improve the visualization of the results, only the brightness and contrast of the images were adjusted, with no further manipulation of the photographs.

\section{RESULTS}

Two parameters were evaluated to study the efficacy of the GEMSP in a chronic EAE model: the abolition of EAE crises and brain leukocyte infiltration as a signal of inflammation.

\section{Clinical Score}

Several factors must be taken into account when using EAE models. Among them, one important point is the clinical score of the animals and their evolution. In our study, the animals were weighed and observed daily (six days a week) by different researchers to preserve objectivity. Three animals included in group 2 (EAE-immunized rats treated with $\mathrm{NaCl}$ ) did not develop EAE correctly. These animals were excluded from the data analysis since no leukocyte infiltration was found, hence corroborating the absence of a clinical score. The remaining animals belonging to group 2 had a specific profile, since the immunological response found in each animal was individual and specific to it (for examples, see Figure 2). Accordingly, in view of the considerable variability between animals, we classified the rats in the second group into three subgroups according to their clinical signs.

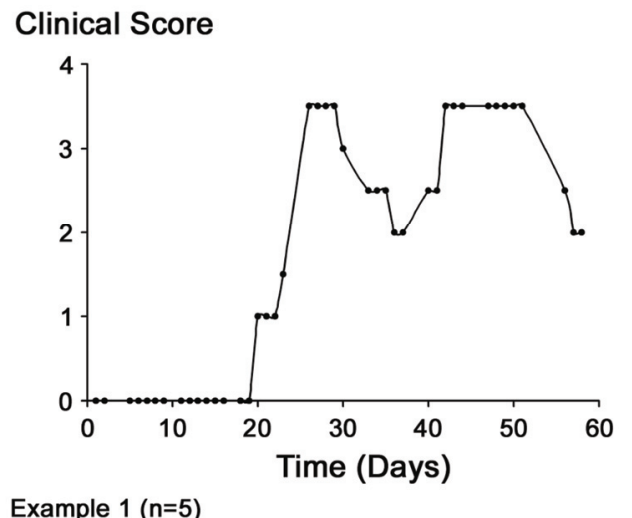

Example $1(n=5)$
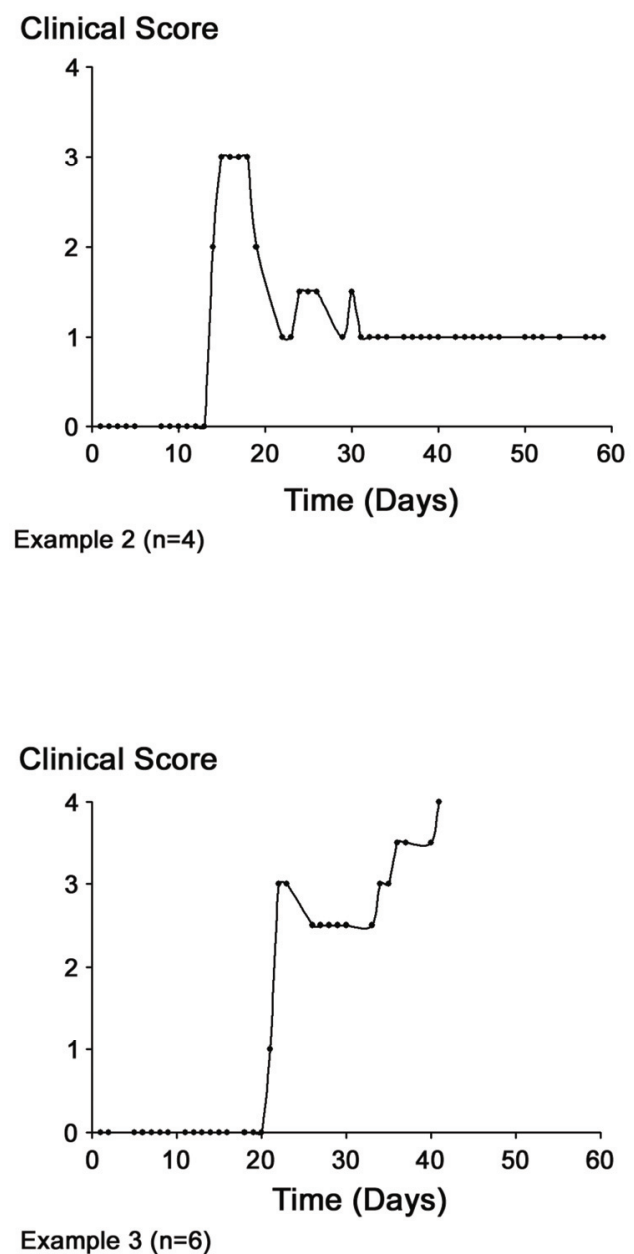

FIGURE 2. Different clinical types. The clinical types observed during the EAE episodes are represented in this figure by three different examples of evolution. These three profiles can be considered to be representative of the clinical stages observed in animals, and can be classified into three subgroups within the group 2 (EAE-immunized rats treated with $\mathrm{NaCl}$ ). n: number of animals. Note that the clinical score of experimental groups 1 and 3 was always 0 (not shown). 
Figure 2 offers examples of three different subgroups of animals with very different clinical patterns (the number of animals showing a similar pattern is indicated).

Neither the animals in group 1 (EAE-immunized rats treated with GEMSP) nor those in group 3 (non-EAE-immunized rats treated with $\mathrm{NaCl}$ ) (control) showed clinical signs. This means that none of the animals in either group developed EAE (Figure 3). This was expected in group 3 because the animals had not been subjected to EAE induction. However, the group 1 animals were induced with the same solution as the group 2 animals, indicating that the absence of EAE in group 1 must been due to the GEMSP treatment.

\section{45 (pan-leukocyte marker)}

\section{Group 1: EAE animals treated with GEMSP}

The results found for this marker in group 1 were in accordance with those previously published in the acute EAE model (see [8]), since in general an absence of brain leukocyte infiltration, or only a very low level, was observed in this group (see Figures $4 \mathrm{~A}$ and $5 \mathrm{~A}$ and Table 3).
TABLE 3. Distribution of brain leukocyte infiltrates in the experimental groups. Leukocyte infiltration visualized with the panleukocyte marker (CD 45) in several brain levels and the spinal cord. -: absence of leukocytes; +: low density of leukocytes; +++: high density of leukocytes; B: Bregma level corresponding to the stereotaxic atlas of Paxinos and Watson (1982); CNS: Central Nervous System; S: scarce distribution (only in some regions); W: widespread distribution in different regions of the level marked.*: region not studied in this experiment.

\begin{tabular}{|c|c|c|c|c|c|c|}
\hline \multirow{3}{*}{$\begin{array}{l}\text { CNS } \\
\text { LEVEL }\end{array}$} & \multicolumn{6}{|c|}{ GROUP } \\
\hline & \multirow{2}{*}{$\begin{array}{c}\text { EAE } \\
\text { RATS } \\
\text { treated } \\
\text { with } \\
\text { GEMSP }\end{array}$} & \multirow{2}{*}{$\begin{array}{c}\text { NON-EAE } \\
\text { RATS } \\
\text { treated with } \\
\text { NaCl }\end{array}$} & \multicolumn{4}{|c|}{ EAE RATS treated with $\mathrm{NaCl}$} \\
\hline & & & D 15 & D 24 & D 60 & $\begin{array}{c}\text { D } \\
100\end{array}$ \\
\hline $\begin{array}{c}\mathrm{B}-0.2 \text { to } \mathrm{B} \\
-0.3\end{array}$ & - & - & $+++/ \mathrm{W}$ & $+/ \mathrm{W}$ & - & - \\
\hline $\begin{array}{c}\mathrm{B}-0.3 \text { to } \mathrm{B} \\
-0.8\end{array}$ & - & - & $+++/ \mathrm{W}$ & $+/ \mathrm{W}$ & $+/ S$ & - \\
\hline B - 1.3 & - & - & $+++/ W$ & * & $+/ S$ & - \\
\hline B - 2.8 & $+/ S$ & - & $+++/ W$ & $+/ \mathrm{W}$ & - & - \\
\hline B - 3.3 & - & - & $+++/ W$ & * & - & - \\
\hline $\begin{array}{c}\mathrm{B}-3.3 \text { to } \mathrm{B} \\
-3.8\end{array}$ & $+/ S$ & - & $+++/ \mathrm{W}$ & * & - & - \\
\hline B - 10.8 & - & - & $+++/ W$ & $+/ W$ & - & - \\
\hline $\begin{array}{c}\mathrm{B}-11.3 \text { to } \\
\mathrm{B}-11.8\end{array}$ & - & - & $+++/ \mathrm{W}$ & $+/ W$ & & - \\
\hline B - 14.3 & - & - & $+++/ W$ & $+/ W$ & - & $+/ S$ \\
\hline $\begin{array}{l}\text { Spinal } \\
\text { Cord }\end{array}$ & - & - & * & * & $\begin{array}{l}+/ S \text { or } \\
+++/ S\end{array}$ & $+/ S$ \\
\hline
\end{tabular}

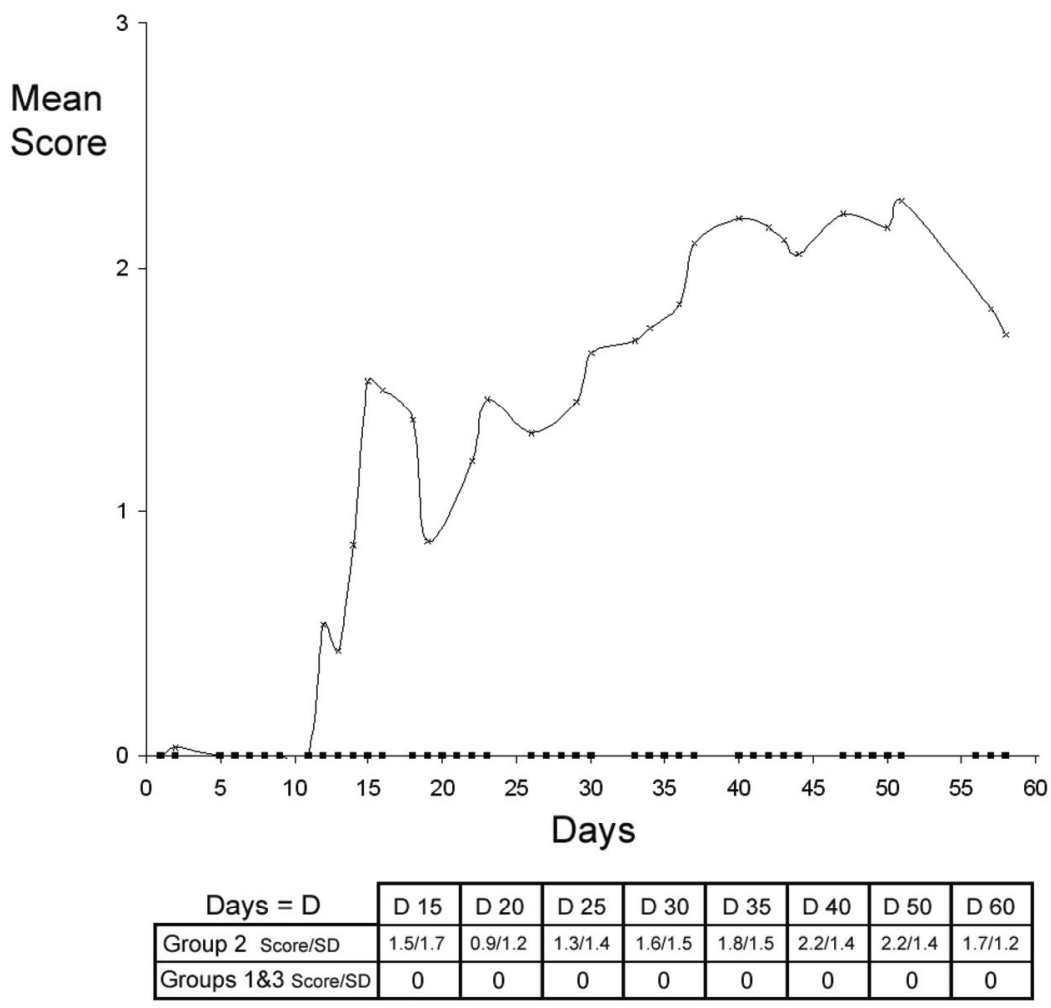

FIGURE 3. Mean clinical scores of the experimental groups (1, 2 and 3). Squares indicate the values of the animals belonging to experimental groups 1 and 3 (clinical score: 0). Note that only animals of group 2 (x) had a clinical score. In the inserted table, the mean clinical score (score) and the standard deviation (SD) are also represented for the three experimental groups at different dates. 

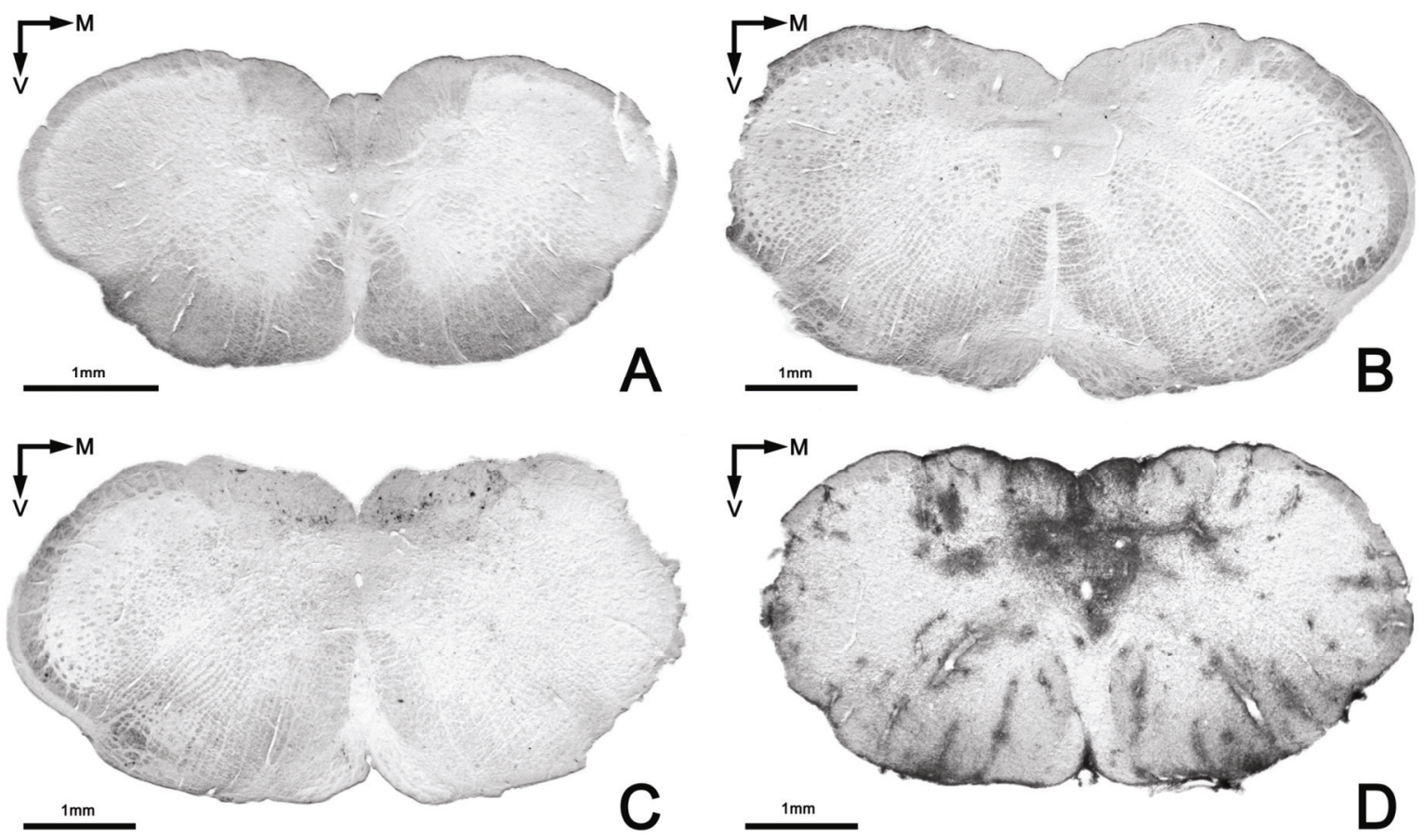

FIGURE 4. Brain leukocyte infiltration in the three experimental groups. Absence of infiltration (anti-CD 45) in the group treated with GEMSP (Group 1) (A) and in the control group (Group 3) (B); C: representative section belonging to animals of group 2 perfused sixty days after induction (D60, see Figure 1); D: section of an animal of group 2 perfused twenty-four days after induction (D24, see Figure 1). Note the marked differences in the infiltration shown in figures 3C (D60) and D (D24); images of the same group showing differences in the process, depending on the perfusion date.
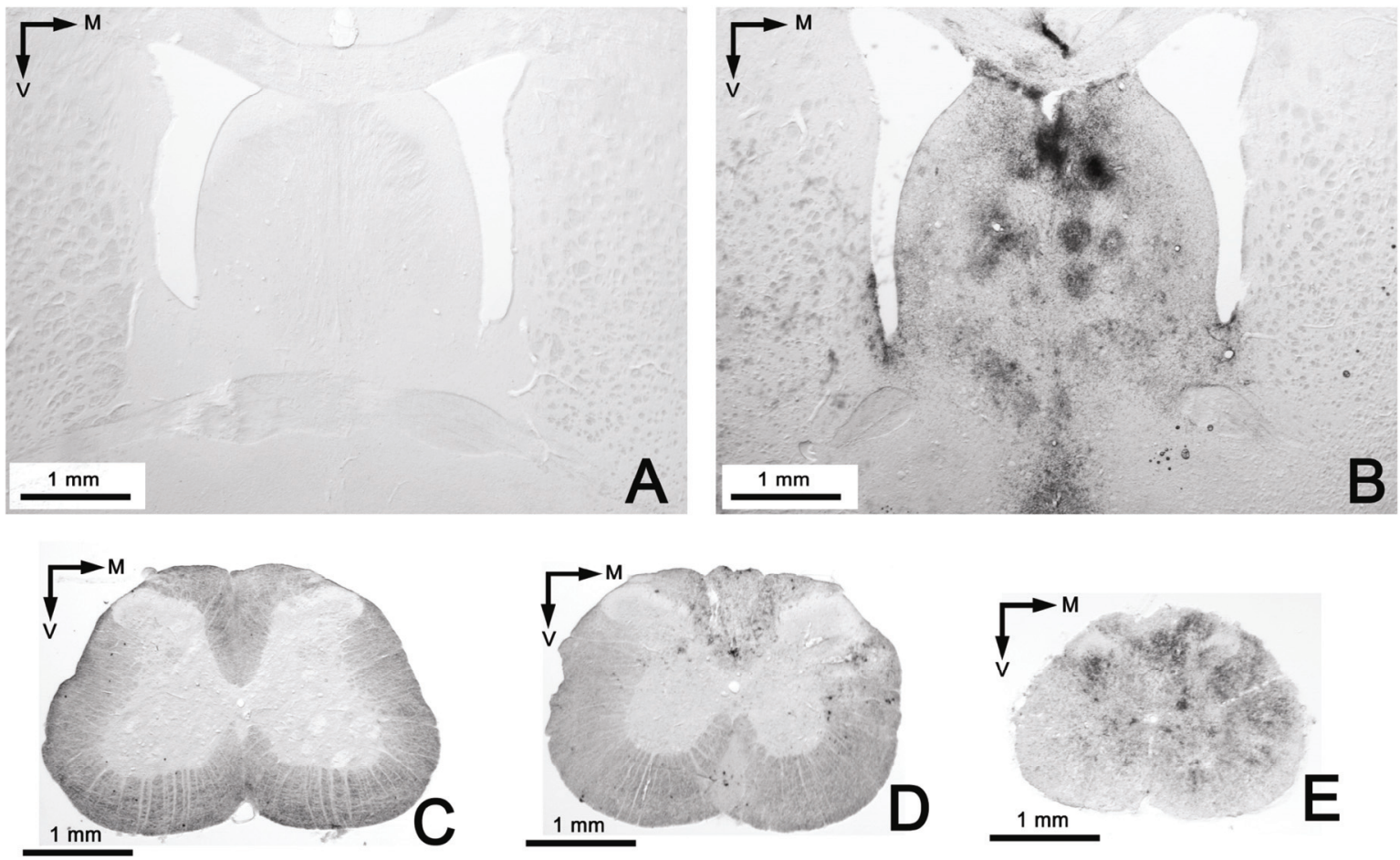

FIGURE 5. Examples of brain and spinal cord leukocyte infiltration visualized with the anti-CD 45 marker. A: Image of the GEMSP group (Group 1), with the absence of leukocyte infiltration in the septum; B: widespread leukocyte infiltration in the septum of group 2 (in the first phases D15-24); C: representative image of group 3 (control: non-EAE $+\mathrm{NaCl}$ ) at spinal cord level, with the absence of leukocyte infiltrates; D: Low-power image of the spinal cord of group 2; E: image of the spinal cord of animals belonging to group 2, at a different level than the image shown in picture D. Note that the infiltrates are much more widespread at this level (E) than at the level shown in D (both pictures belong to animals perfused on D60). 


\section{Group 2: EAE animals treated with $\mathrm{NaCl}$}

We observed that the animals in the first stages of the EAE episode (around two weeks after immunization, D15, and three-four weeks after immunization, D24) (see Figure 1) had developed widespread and strong leukocyte infiltration in the CNS (Figures 4D, 5B, and Table 3); this infiltration was even higher than that previously described in the acute EAE model [8]. However, in later stages (D60 and D100) (see Figure 1) leukocyte infiltration decreased, although the clinical signs persisted (see Figures 2, 4C and Table 3). Thus, as may be seen in Figure 2, the clinical signs still remained after a fairly long period of time. This suggests that the degree of infiltration was not correlated with the clinical signs, since the degree of infiltration in the animals of group 2 differed, depending on the different stages (see Figures 4C, D, and Table 3). Similar data have been reported previously for the acute EAE model [8]. Only some animals continued to display a low leukocyte infiltration in certain localized regions at D60 (see Table 3), especially in lower brain levels and the spinal cord (see Figure 5D and E).

\section{Group 3: control}

No brain leukocyte infiltration was found in the animals of group 3 (see Figures 4B, 5C and Table 3).

\section{GEMSP in the central nervous system}

Following application of

FIGURE 6. Immunoreactivity for GEMSP. Immunoreactivity located in the ventral horn motoneurons of the rat spinal cord was visualized only in the animals treated with GEMSP (A and B, first group). The immunoreactivity disappeared in animals of the first group when preabsorption (C) and elimination (D) of the first antibody (anti-conjugated methionine) were carried out. Immunoreactivity was absent in the animals of the second $(\mathrm{EAE}+\mathrm{NaCl})(\mathrm{E})$ and third groups (control: non-EAE + NaCl) $(\mathrm{F})$. the anti-conjugated methionine antibody, we observed immunoreactivity in the motoneurons located in layer IX of the ventral horn along the whole spinal cord of the animals belonging to group 1 (treated with GEMSP) (Figure 6A, B). However, in this first group, after carrying out the immunocytochemical histological controls (preabsorption of the first antibody (Figure 6C) and elimination of the first antibody (Figure 6D)), in both cases we failed to observe immunoreactivity in those neurons. In addition, in these ventral horn motoneurons of the rat spinal cord we did not observe any immunoreactivity towards conjugated methionine in the animals of either the second (Figure 6E) or the third (control, Figure $6 \mathrm{~F}$ ) experimental groups. In both latter groups, the animals were not treated with GEMSP. Finally, it should be noted that in the brain we did not observe immunoreactivity towards GEMSP in any of the animals belonging to the three experimental groups.
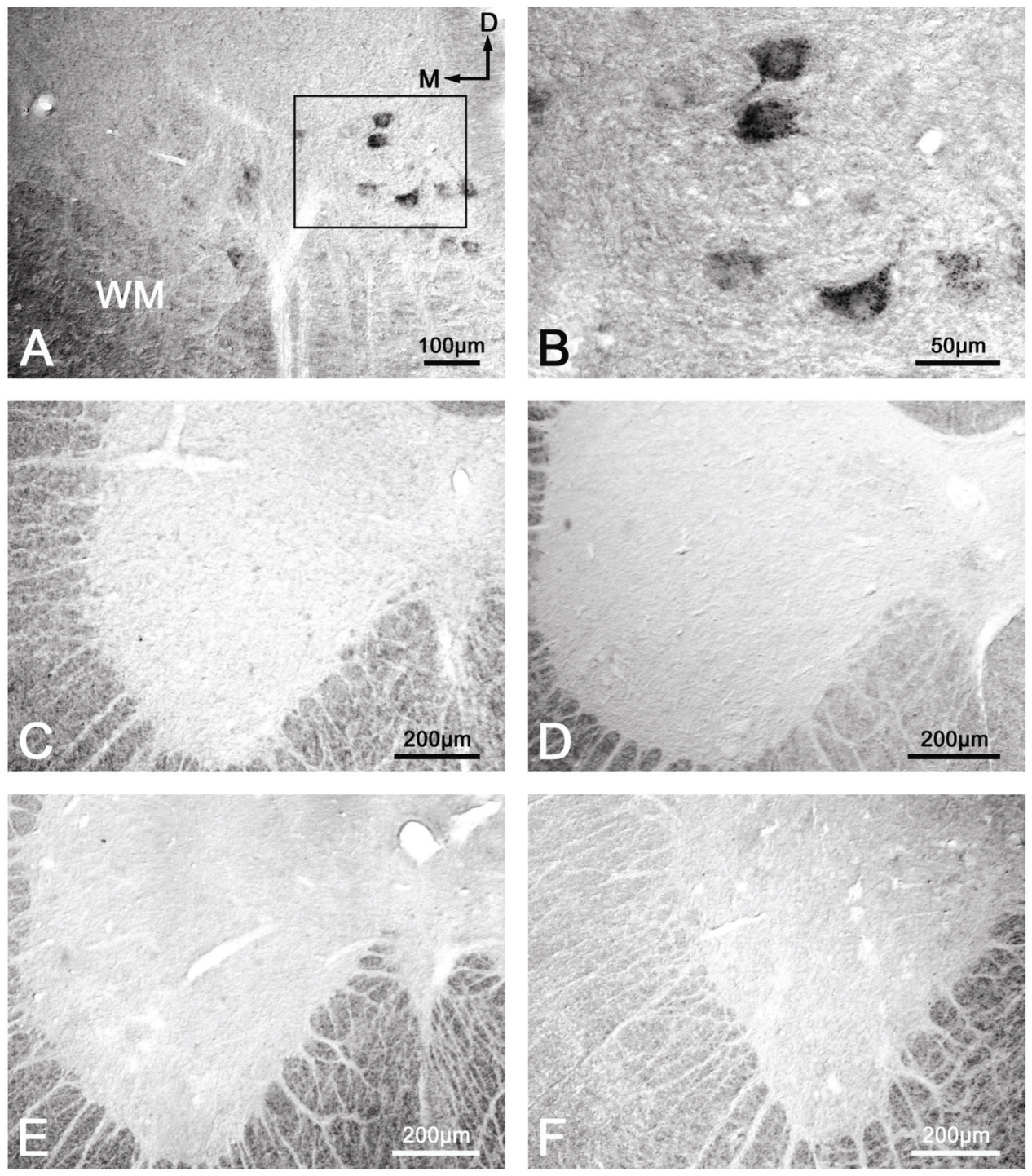


\section{DISCUSSION}

Here, we report for the first time that GEMSP abolishes the development of chronic EAE; that the GEMSP is stored inside nerve cells, and that, in a chronic model of EAE, GEMSP abolishes brain leukocyte infiltration.

\section{Effects of the GEMSP dose on EAE}

It has previously been reported [8] that the administration of low doses ( $0.75 \mathrm{mg}$ per day) of GEMSP reduces brain leukocyte infiltration to a considerable extent, although no effect against the development of EAE was observed at such a dose. In order to determine the lowest dose with the maximum effect, we carried out a dose-range study in acute and chronic EAE models to determine whether there were other effective doses that might abolish EAE crises (unpublished data). The results of those experiments showed that the dose used here (7.5 $\mathrm{mg}$ per day) seems to be the most effective for abolishing acute and chronic EAE episodes. In this sense, we observed that there was a true dose-range effect on EAE clinical attack, since lower doses ( $3.75 \mathrm{mg}$ per day) were not as effective in abolishing EAE crises, and doses of 0.75 $\mathrm{mg} /$ day and $1.875 \mathrm{mg} /$ day were not effective at all against EAE crise (see [8]). At a dose of $3.75 \mathrm{mg}$, GEMSP seems to exert a strong reduction of EAE episodes, although not a complete abolition in the acute EAE model. Here, we used a dose ten times higher $(7.5 \mathrm{mg})$ than in the previous study [8] to see whether GEMSP completely abolishes the EAE process or not. The results reported here show for the first time that long-term treatment with GEMSP completely abolishes the development of EAE in a chronic model of the disease.

\section{Effects of GEMSP on Brain Leukocyte Infiltration and Clinical Scores}

As mentioned above, brain leukocyte infiltration related to inflammation has previously been evaluated using different drugs in an EAE model [3, 5, 6, 8-11]. However, only the study by Mangas et al. [8] reported the action of GEMSP and offered details about the neuroanatomical distribution of brain leukocyte infiltration, although in that study no data on demyelization were provided because the investigation was carried out in an acute EAE model. In both acute and chronic EAE models, the induced animals exhibited a very strong degree of leukocyte infiltration throughout their brains. The animals with acute EAE perfused at the peak of the EAE episode displayed a pronounced and widespread brain leukocyte infiltration [8]. This observation was similar to the results seen in animals with chronic EAE perfused at the peak of the first EAE clinical attack or episode. The first EAE clinical attack in the chronic EAE model was comparable to the peak of acute EAE, since the degree of leukocyte infiltration was similar. Thus, both models revealed very strong and widespread brain infiltration, although leukocyte infiltration seemed to be higher at this stage (first clinical attack) in chronic EAE in comparison with previously reported data found for acute EAE.

Previously published data have shown that the administration of GEMSP (0.75 mg per day) is sufficient to significantly decrease brain leukocyte infiltration, even though the EAE episode is not modified with this low dose of GEMSP [8]. In the present work, after the administration of GEMSP (7.5 mg per day) to animals in which a chronic model of EAE was developed the dose was also effective against leukocyte brain infiltration.

As mentioned in the Results section, our data clearly show that there was no correlation between the clinical score and leukocyte infiltration, these data are in accordance with previously published results for acute EAE [8]. In the present work, we observed the crucial importance of leukocyte infiltration during the first stages on EAE, a mechanism that could play a secondary role in ensuing stages, as occurs in MS. This is very important for establishing the efficiency of current therapies for MS. Such therapies are focused on immunomodulation (GA and IFN- $\beta$ ), and such drugs only treat this aspect of the disease.

The variable clinical signs in each animal belonging to group 2 (EAE animals treated with $\mathrm{NaCl}$ ), as well as the score and evolution of the disease in the animals of the same group, indicates the complexity of this chronic EAE model. Figure 2 shows the different rat profiles. The variability observed in this chronic model reflects the complexity of the immune system responses. This variability is similar in MS patients. Accordingly, each animal or patient must be considered as a single immune entity.

Moreover, current therapies against MS elicit a large number of well-reported side effects [1]. The side effects of IFN $\beta$ are injection-site reaction, flu-like symptoms, depression, elevated liver enzymes, and leukopenia [1], while those for glatiramer acetate are injection-site reaction, postinjection systemic reactions, and chest tightness or pain and lymphadenopathy [1]. However, to date no side effects have been reported for the new potential drug addressed here (GEMSP), either in an open clinical trial or in experimental animals. This is because GEMSP does not elicit either biological, haematological, or hepatic side effects [8]. Finally, we are currently unaware of whether the effect of GEMSP on leukocyte infiltration is due to the fact that the blood-brain barrier has been repaired and/or 
whether this new drug candidate might diminish the effect of immune mediators or have some immunomodulatory function. This aspect should be addressed in future studies.

\section{GEMSP inside motoneurons}

Our findings show that some components of GEMSP cross the blood-brain barrier and that the drug is stored inside the motoneurons of the ventral horn of the spinal cord. This suggests that GEMSP could enter the motoneurons by means of pinocytosis or endocytosis, since it is well known that PL (to which the methionine is conjugated in GEMSP) is introduced into the cytosol by those mechanisms [21]. It therefore seems that the localization of the component (conjugated methionine) of GEMSP inside the motoneurons could be explained in terms of PL transport into the cell, although specific receptors for some of the components of the GEMSP might be involved in the incorporation of such components into the motoneurons. Moreover, motoneurons are well known to be rich in methionine residues, although conjugated methionine cannot be used for the synthesis of peptides because here it was conjugated to PL via glutaraldehyde. At present, we are unaware of the physiological consequences of the morphological findings observed here. Nevertheless, it is known that vitamins and thiols (glutathione, cysteine, methionine...) are involved in the neutralization of free radicals, inhibiting apoptotic mechanisms [22-24]. This could be one of the possible actions of GEMSP on motoneurons. Previous pharmacokinetic studies carried out by our group have shown that the components of the GEMSP can be detected in serum at least $24 \mathrm{~h}$ after a single subcutaneous injection of GEMSP. Moreover, a serum concentration peak of the components of GEMSP was found two hours after the administration of GEMSP. Despite this, we are unable to explain why GEMSP was only found in the spinal cord and not in the brain of the animals of the group 1 . In this sense, it is possible that GEMSP could be metabolized faster in the brain than in the spinal cord.

The antibodies used here for the localization of one component (methionine-G-PL) of GEMSP were generated with an immunogen containing a carrier protein (bovine serum albumin, BSA) other than PL, since it is known that PL is a non-immunogenic peptide (see patent numbers of GEMSP: 6114388 (USA) and 792167 (EU)). However, it should be remarked that according to ELISA, the anti-conjugated methionine antibodies recognized both the methionine conjugated via glutaraldehyde to BSA and that conjugated to PL. These data were confirmed when the preabsorption of the diluted antibody (anti-conjugated methionine) was carried out with methionine-G-BSA or with methionine-G-PL (a component of GEMSP). In both cases, the immunoreactivity observed in the motoneurons disappeared. This means that the antibody specifically recognizes the same part of the antigen (methionine-G-first lysine residue), since this lysine residue is present in both BSA and PL. As has been previously demonstrated, it is known that free methionine is not recognized by the antibody used in the present study (anti-conjugated methionine) [25], and the same is the case of other molecules (e.g., acetylcholine, tryptophan, 5-hydroxytryptophan, 5-hydroxytryptamine, 5-methoxytryptophan and 5-methoxytryptamine) conjugated in the same way as the methionine studied here [26, 27]. It is also known that paraformaldehyde does not fix free molecules such as amino acids and their derivatives quickly, unlike glutaraldehyde. Here, we perfused the animals with paraformaldehyde, and hence the free methionine was not fixed, whereas the methionine (non-free)-G-PL was specifically recognized by the antibodies directed against the conjugated methionine via glutaraldehyde. It is also known that when methionine is conjugated via other coupling agents (e.g., glutaric anhydride) it is not recognized by these antibodies (see [25]). Using paraformaldehyde, methionine-G-PL was fixed to the tissue by the free amines of the PL, this being due to the free amine groups not linked to the methionine (more than $50 \%$ of the residues of PL are still free after linkage to methionine via glutaraldehyde). Additionally, the competition experiments carried out with ELISA showed that the antibodies were specific to conjugated methionine and did not recognize other molecules (see Table 2). All the above data indicate that the observed immunoreactivity is specific to one component (methionine-G-PL) of the GEMSP.

It should be noted that methionine-G-BSA cannot be included as a component of GEMSP: first, because of the animal origin of BSA (this compound could induce possible unexpected side effects (e.g., allergy...) that cannot be controlled) and second, because if BSA is used antibody production against the drug will be induced and this could neutralize the therapy and produce important side effects. Accordingly, we used PL instead of BSA in the development of this potential therapy (GEMSP).

\section{Available Data on GEMSP Toxicity}

The toxicity of GEMSP has been tested by Gemacbio (Cenon, France) as well as by an independent laboratory (EVIC, France) (see [8]). The results reveal zero mortality in animals after a single intravenous dose of $10 \mathrm{mg} / \mathrm{kg}$ (LD0 and LD50 >10 $\mathrm{mg} / \mathrm{kg}$ ) (see [8]). The quantities used in the toxicity study were lower than those used in the present study, 
but here the product was administered subcutaneously.

\section{Plausible Therapeutic Window of GEMSP in MS patients}

The available clinical data show that the key inclusion criteria for the therapeutic window in humans in an open clinical trial would be: a) disability, as evaluated by the expanded disability status scale (EDSS) in patients with Secondary Progressive Multiple Sclerosis (SPMS) (EDSS 3-6.5); b) a minimum 1-point increase in EDSS during the 24 months prior to the beginning of the clinical trial. The results of the statistical analysis obtained at six months of treatment with low doses of GEMSP $(0.75 \mathrm{mg} /$ day $)$ were conclusive: $55 \%$ of the patients maintained a stable EDSS value and $18 \%$ of patients had decreased EDSS values [8], instead of a normal progression of 0.25 points on the mean EDSS scale. These data allow us to conclude that GEMSP could be an effective new drug candidate for MS treatment as shown in the acute EAE model [8] and in the present study carried out with a chronic EAE model.

In sum, the activity of GEMSP against leukocyte infiltration previously shown in the acute EAE model has been validated in the chronic EAE model. Moreover, we found that there was no correlation between the degree of EAE and leukocyte infiltration. Our results demonstrate the presence of GEMSP (methionine-G-PL) in the motoneurons of the spinal cord, and the drug appears to be a good candidate for the prevention and treatment of MS, since GEMSP completely abolished the crises and the clinical scores in a chronic EAE model. The finding of an anatomical location of GEMSP in the spinal cord opens new lines of research (electron microscopy, electrophysiology, brain slices...) to gain further insight into the action of the drug on motoneurons (for example, it is quite important to know whether GEMSP acts on gene regulation, and specifically on the expression of growth factors; to determine the possible action of GEMSP, by neutralizing free radicals, on the inhibition of apoptotic mechanisms...). Moreover, a previous work [8] has shown that some amino acids and their derivatives (methionine, cysteine, taurine and 5-methoxy-tryptamine) play an important role as radical scavengers and as neuroprotective components. These molecules, including methionine, are linked to PL and are components of GEMSP. In the future, other possible actions of the GEMSP, such as anti-oxidant and anti-radical stresses and axonal protection, should be investigated in depth, but the data reported here do suggest that GEMSP may be considered as a new potential drug candidate for the treatment of MS.

\section{ACKNOWLEDGEMENTS}

This work has been supported by Gemacbio (Cenon, France), the Institut pour le Developpement de la Recherche en Pathologie Humaine et Therapeutique (IDRPHT) (Talence, France), Red de Terapia Celular de Castilla y León (Spain), Consejería de Educación de la Junta de Castilla y León (Spain) and the INCYL-Federación de Cajas de Ahorro de Castilla y León (Spain). The authors wish to thank to N. Skinner for supervising the English text.

\section{CONFLICT OF INTEREST}

The authors have declared that no conflict of interest exists.

\section{REFERENCES}

1. Langer-Gould A, Moses HH, Murray TJ. Strategies for managing the side effects of treatments for multiple sclerosis. Neurology 2004; 63: S35-S41

2. Lehmann D, Karussis DM, Fluresco D, et al. Immunomodulation of autoimmunity by linomide: inhibition of antigen presentation through down regulation of macrophage activity in the model of experimental autoimmune encephalomyelitis. J Neuroimmunol 1997; 74: 102-110.

3. Van der Meide PH, de Labie MC, Ruuls SR, et al. Discontinuation of treatment with IFN- $\beta$ leads to exacerbation of experimental autoimmune encephalomyelitis in Lewis rats. Rapid reversal of the antiproliferative activity of IFN- $\beta$ and excessive expansion of autoreactive $\mathrm{T}$ cells as disease promoting mechanisms. J Neuroimmunol 1998; 84: 14-23.

4. Aharoni R, Teitelbaum D, Leitner O, et al. Specific Th2 cells accumulate in the central nervous system of mice protected against experimental autoimmune encephalomyelitis by copolymer 1. Proc Natl Acad Sci USA 2000; 97: 11472-11477.

5. Brunmark C, Runström A, Ohlsson L, et al. The new orally active immunoregulator laquinimod (ABR-215062) effectively inhibits development and relapses of experimental autoimmune encephalomyelitis. J Neuroimmunol 2002; 130: 163-172.

6. Floris S, Ruuls SR, Wierinckx A, et al. Interferon- $\beta$ directly influences monocyte infiltration into the central nervous system. J Neuroimmunol 2002; 127: 69-79.

7. Teitelbaum D, Aharoni R, Klinger E, et al. Oral glatiramer acetate in experimental autoimmune encephalomyelitis: clinical and immunological studies. Ann NY Acad Sci 2004; 1029: 239-249.

8. Mangas A, Coveñas R, Bodet D, et al. Evaluation of the effects of a new drug on brain leukocyte infiltration in an experimental model of autoimmune encephalomyelitis. Lett Drug Des Discovery 2006; 3: 138-148.

9. Yu M, Nishiyama A, Trapp BD, et al. Interferon- $\beta$ inhibits progression of relapsing-remitting experimental autoimmune encephalomyelitis. J Neuroimmunol 1996; 64: 91-100.

10. Karussis DM, Lehmann D, Slavin S, et al. Treatment of chronic-relapsing experimental autoimmune encephalomyelitis with the synthetic immunomodulator linomide (quinoline-3-carboxamide). Proc Natl Acad Sci USA 1993; 90: 6400-6404.

11. Nguyen KB, McCombe PA, Pender MP. Increased apoptosis of T lymphocytes and macrophages in the central and peripheral nervous systems of Lewis rats with experimental autoimmune encephalomyelitis treated with dexamethasone. J Neuropathol Exp Neurol 1997; 56: 58-69.

12. Noseworthy JH, Lucchinetti C, Rodríguez M, et al. Multiple Sclerosis. N Engl J Med 2000; 343: 938-952.

13. Kappos L, Moeri D, Radue EW, et al. Predictive value of gadolinium-enhanced magnetic resonance imaging for relapse rate and changes in disability or impairment in multiple sclerosis: a 
meta-analysis. Gadolinium MRI Meta-analysis Group. Lancet 1999; 353: 964-969.

14. Weissert R, Wallström E, Storch MK, et al. MHC Haplotype-dependent regulation of MOG-induced EAE in rats. J Clin Invest 1998; 102: 1265-1273.

15. Villoslada P, Abel K, Heald N, et al. Frequency, heterogeneity and encephalitogenicity of $\mathrm{T}$ cells specific for myelin oligodendrocyte glycoprotein in naive outbred primates. Eur J Immunol 2001; 31: 2942-2950.

16. Mangas A, Coveñas R, Geffard K, et al. Folic acid in the monkey brain: an immunocytochemical study. Neurosci Lett 2004; 362: 258-261.

17. Mangas A, Coveñas R, Geffard K, et al. Thiamine-like fibers in the monkey brain: An immunocytochemical study. Life Sci 2006; 79 : 1121-1128.

18. Mangas A, Coveñas $\mathrm{R}$, Geffard $\mathrm{K}$, et al. Riboflavin-like immunoreactive fibers in the monkey brain. Anat Embryol 2006; 211: 267-272.

19. Mangas A, Coveñas R, Bodet D, et al. Immunocytochemical visualization of D-Glutamate in the rat brain. Neuroscience 2007; 144: 654-664.

20. Paxinos G., Watson C. The Rat Brain in Sterotaxic Coordinates. Sydney, Australia: Academic Press; 1982.

21. Ryser H J, Shen WC. Conjugation of methotrexate to poly-(L-lysine) increases drug transport and overcomes drug resistance in cultured cells. Proc Natl Acad Sci USA 1978; 75: 3867-3870.

22. Helgestad J, Storm-Mathisen I, Lie SO. Vitamin C and thiol reagents promote the in vitro growth of murine granulocyte/macrophage progenitor cells by neutralizing endogenous inhibitor(s). Blut 1986; 52: 1-8.

23. Iwata-Ichikawa E, Kondo Y, Miyazaki I, et al. Glial cells protect neurons against oxidative stress via transcriptional up-regulation of the glutathione synthesis. J Neurochem 1999; 72: 2334-2344.

24. Halliwell B. Reactive species and antioxidants. Redox biology is a fundamental theme of aerobic life. Plant Physiol 2006; 141: 312-322.

25. Amara A, Coussemacq M, Geffard M. Molecular detection of methionine in rat brain using specific antibodies. Neurosci Lett 1995; 185: 147-150.

26. Geffard M, Dulluc J, Rock A. Antisera against the indolealkylamines: tryptophan, 5-hydroxytryptophan, 5-hydroxytryptamine, 5-methoxytryptophan and 5-methoxytryptamine tested by an enzyme-linked immunosorbent assay method. J Neurochem 1985; 44: 1221-1228.

27. Geffard M, McRae-Degueurce A, Souan ML. Immunocytochemical detection of acetylcholine in the rat central nervous system. Science 1985; 229: 77-79. 